\title{
Single-image and double-image holographic coupler/optical fiber: a comparative study
}

\author{
Maria Luisa Calvo and Maria Lourdes de Pedraza
}

\begin{abstract}
A mathematical model that describes a fiber-to-fiber holographic connector is presented. We consider both single- and double-image cases for the reconstruction of the second hologram. A comparative study of the two coupling conditions is presented. Fourier analysis of the coupling equation enables us to study the linearity of the system.

Key words: Holocoupler, optical interconnections, coupling in optical fibers.
\end{abstract}

\section{Introduction}

In the past decade considerable work has been done on the development of techniques for optical interconnects and networks based on holographical components. ${ }^{1,2}$ Applications of this technology include (but are not limited to) optoelectronic devices, biomedical optical fibers, and optical arrays for optical computing. ${ }^{3-5}$ There is a need for mathematical models that describe the behavior of these optical systems. To characterize a holographic interconnector, theories on the propagation of electromagnetic fields in optical waveguides and diffraction of light by volume holograms must be combined. Our aim is to emphasize the optimizing conditions for high diffraction efficiency in a holocoupler/optical fiber system. The first theoretical model, which was developed by Soares, ${ }^{6}$ was based on a scalar treatment for the object wave under Fresnel diffraction conditions. Here, an off-axis hologram was recorded, and a single-image (single-focus) reconstruction was obtained. The coupling conditions of this system are analyzed in this paper. We show the feasibility of coupling light into a second fiber under less restrictive conditions. In a previous paper, ${ }^{7}$ we developed a model for the holocoupling system with an on-axis recording (double focus) and introduced the field that was scattered by the fiber excited by lateral illumina-

M. L. Calvo is with the Departamento de Optica, Facultad de Ciencias Físicas, Universidad Complutense de Madrid, Madrid 28040, Spain. M. L. de Pedraza is with the Departamento de Enfermería II, Escuela Universitaria de Enfermería, Fisioterapia y Podología, Universidad Complutense de Madrid, Madrid 28040, Spain.

Received 31 July 1991.

0003-6935/93/213969-09\$06.00/0.

(C) 1993 Optical Society of America. tion as the TM-polarized object wave. The optimization parameters were deduced from the analysis of the coupling conditions, namely, refractive-index distribution of the optical fiber, polarization state of the reference wave, geometric configuration of the object wave, angle of incidence, and the absorption coefficient of the photomaterial. ${ }^{8}$ In this paper we undertake a comparative study of single- and double-image recording procedures and analyze the linear behavior of the second case by applying the Fourier-transform operator to the coupling equation. This analysis in the spatial frequency domain provides information on the amount of energy processed in the holocoupling procedure. Since the linearity is assured, the losses of energy could be the result of the misalignement of the system and the influence of the optical properties of the photomaterial (absorption). This paper is organized as follows: Section 2 is dedicated to the single-image (off-axis) coupling condition based on the experimental setup of Leite et al. ${ }^{9}$ The numerical estimates show that the coupling holds in the core of the fiber for particular bound modes inside it. In Section 3 we formulate a second coupling condition by considering a double-image recording (on-axis hologram), and assuming some modifications in the experimental setup. The solution is given by a pair of implicit equations. The numerical results exhibit less restrictive conditions than in the single-image case. In Section 3 we test the linear behavior of the double-image device by applying Fourier-transform techniques.

\section{Single-Image Holocoupler Recording: Coupling Conditions}

As shown in Fig. 1, the recording of the holocoupler is achieved by interfering the object wave, a scattering field coming from the output face of the first optical 


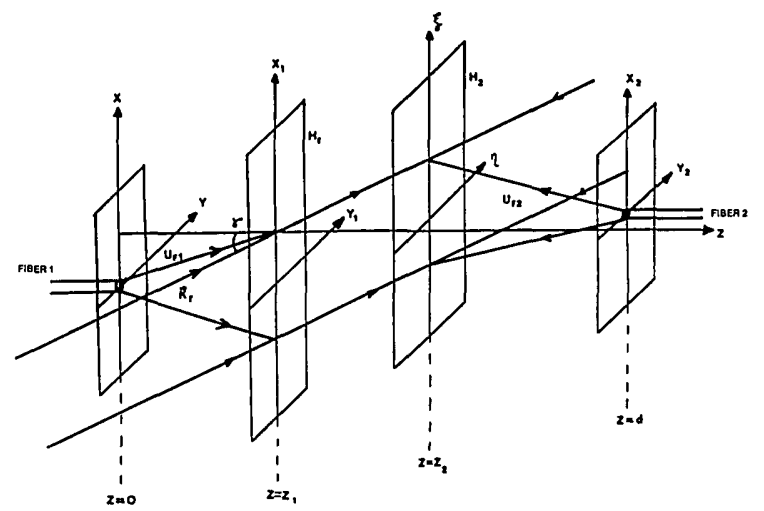

Fig. 1. Schematic representation of the experimental setup for recording (above) and reconstruction (below) of an off-axis holocoupler.

fiber, and a monochromatic plane-polarized reference wave in a first holographic plate. A second recording is performed under similar conditions, but the first reference wave is replaced by its conjugate. The absence of losses in the photomaterial and linear recording conditions is assumed, and the coupling is produced when the field transmitted by the second hologram equals the conjugate output field of the second fiber. In this paradigm, if a scalar treatment is applied, no variations in the coupling condition that are due to polarization states of the object and reference wave are deduced. The simplification of the modeling comes from the TM mode, which is excited by a lateral illumination of the fiber. Recently experimental results have demonstrated that a lower energy conversion into guided modes inside the fiber takes place, and, the TE mode seems to be more suitable. ${ }^{10}$ The scattering pattern produced by the TM mode shows a nonnegligible intensity distribution. There is also a relative dependence on the geometry of the illuminating wave front, and changes in the spot of the forward-scattered light are observed.

The coupling condition is merely a normalization ${ }^{7}$ :

$$
\left|U_{F 1}\right|^{2}\left|U_{R 1}\right|^{2}=1 \text {. }
$$

Here $U_{F 1}$ is the field scattered by the first fiber, and $U_{R 1}$ represents the reference wave. If we assume a monochromatic plane-polarized reference wave, Eq. (1) can be further simplified to

$$
\left|U_{F 1}\right|^{2}=1 \text {. }
$$

This field can be easily formulated by considering the eikonal approximation, which holds for small-angle scattering. Quantitatively the scattering angle $\theta$ is limited by the approximate condition ${ }^{11}$ (Glauber's approximation),

$$
\theta^{2} K d \leq 1 \text {. }
$$

$K$ represents the wave vector $K=|\mathbf{K}|=2 \pi / \lambda, \lambda$ is the wavelength of the incoming radiation, and $d$ is the fiber diameter. Previous work ${ }^{7}$ shows the large validity of the eikonal approximation for both single-mode and multimode fibers working in the visible and near-IR region, provided that coupling constant $\Delta$ of the fiber obeys the condition $\Delta \leq 1$ (weakly guide approximation). Moreover, if $\mathbf{K}$ is the wave vector of the scattered field, energy conservation requires that $|\mathbf{K}|=\left|\mathbf{K}^{\prime}\right|$, so that, for small-scattering angles, the vector $\mathbf{K}-\mathbf{K}^{\prime}$ is nearly perpendicular to $\mathbf{K}$, which is a restriction to be considered in experimental studies. The restriction could be overcome by the use of the same wave front for the lateral illumination and for the reference wave. Assuming the eikonal approximation, a first result for the normalization condition for the scattered field can be obtained:

$$
2 \cos \varphi_{\text {eik }}\left(X_{2}\right)=1,
$$

where $\varphi_{\text {eik }}$ is the eikonal phase of the scattered field, and $X_{2}$ is the coordinate at the transverse plane of the fiber.

We define $T_{\text {eik }}{ }^{(1)}\left(\mathbf{K}^{\prime}, \mathbf{K}, X_{1}\right)$ as the field scattered by an arbitrary concentric layer $M$ of variable radius $R_{M}=\sqrt{R_{1}^{2}-} X_{1}^{2}$, located at an arbitrary $X_{1}$ point inside the core, as shown in Fig. 2. $T_{\text {eik }}^{(1)}\left(\mathbf{K}^{\prime}, \mathbf{K}, X_{1}\right)$ can be separated into real $\left[T_{\text {eik }}{ }^{(1, r)}\right]$ and imaginary $\left[T_{\text {eik }}^{(1, i)}\right]$ parts:

$$
\begin{aligned}
T_{\mathrm{eik}}^{(1)}\left(\mathbf{K}^{\prime}, \mathbf{K}, X_{1}\right)= & T_{\mathrm{eik}}{ }^{(1, r)}\left(\mathbf{K}^{\prime}, \mathbf{K}, X_{1}\right) \\
& +i T_{\mathrm{eik}}{ }^{(1, i)}\left(\mathbf{K}^{\prime}, \mathbf{K}, X_{1}\right),
\end{aligned}
$$

$T_{\text {eik }}^{(1, r)}\left(\mathbf{K}^{\prime}, \mathbf{K}, X_{1}\right)$

$$
\begin{aligned}
= & \frac{1}{2} \sqrt{\frac{K}{\pi}} \int_{-\sqrt{R_{1}^{2}-X_{1}}}^{\sqrt{R_{1}^{2}-} X_{1}{ }^{2}} \mathrm{~d} X_{2}^{\prime} \cos \left(K X_{2}^{\prime} \sin \theta\right) \\
& \times\left[\cos \varphi_{\text {eik }}\left(X_{2}^{\prime}\right)+\sin \varphi_{\text {eik }}\left(X_{2}^{\prime}\right)-1\right],
\end{aligned}
$$

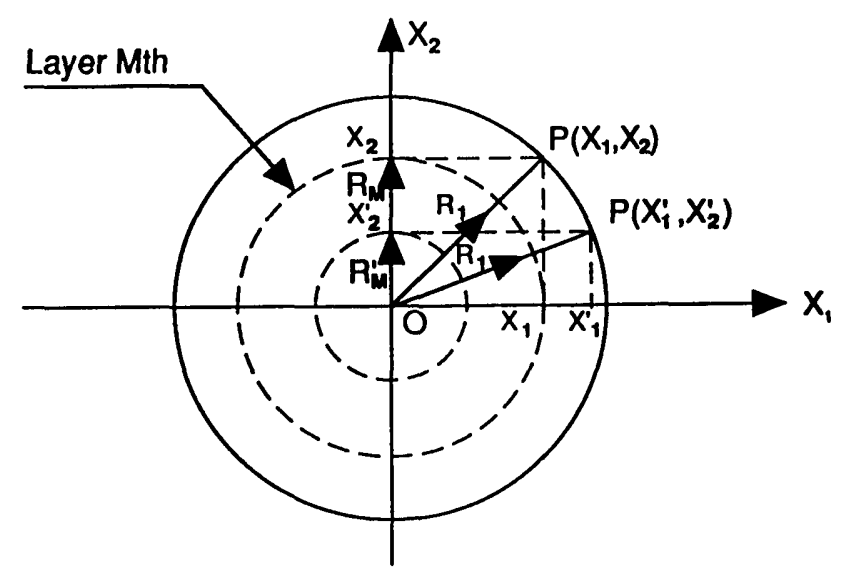

Fig. 2. Definition of variable radius $R_{M}$ for the $M$ th layer: $R_{M}=$ $\left(R_{1}^{2}-X_{1}^{2}\right)^{1 / 2}$ for points inside the nucleus at the cross section of the fiber; $0 \leq R_{M} \leq R_{1}$. The zeroth-order layer is defined for $R_{M}=0\left(X_{1}=R_{1}\right)$. The last-order layer is defined for $R_{M}=R_{1}$ $\left(X_{1}=0\right)$. 
$T_{\text {eik }}^{(1, i)}\left(\mathbf{K}^{\prime}, \mathbf{K}, X_{1}\right)$

$$
\begin{aligned}
& =\frac{1}{2} \sqrt{\frac{K}{\pi}} \int_{-\sqrt{R_{1}^{2}-X_{1}{ }^{2}}}^{\sqrt{R_{1}^{2}-} X_{1}{ }^{2}} \mathrm{~d} X_{2}^{\prime} \cos \left(K X_{2}^{\prime} \sin \theta\right) \\
& \times\left[-\cos \varphi_{\text {eik }}\left(X_{2}{ }^{\prime}\right)+\sin \varphi_{\mathrm{eik}}\left(X_{2}{ }^{\prime}\right)+1\right],
\end{aligned}
$$

where $R_{1}$ is the radius of the core of the fiber, and $X_{1}$ is the coordinate at the transverse plane of the fiber perpendicular to $X_{2}$. In Eqs. (6) and (7) we define $\left[T_{\text {eik }}{ }^{(1, r)}\right]$ and $\left[T_{\text {eik }}{ }^{(1, i)}\right]$ as the real and imaginary parts of $T_{\text {eik }}{ }^{(1)}\left(\mathbf{K}^{\prime}, \mathbf{K}, X_{1}\right)$, respectively, of the sectorial contribution of the scattering field for arbitrary points $X_{1}$ : $0 \leq X_{1} \leq R_{1}$ inside the output face of the fiber. In this case we are not dealing with the classical electromagnetic field (unique dependence on the scattering angle), but the variable integration limits give the distribution of the energy in partial regions of the fiber (in order to appreciate the coupling condition). Therefore we are not strictly speaking of the fiber as a scattering center as in the classical treatment. This model reminds us, in a certain sense, of the one introduced by Cordero Iannarella ${ }^{12}$ for the analysis of scattering of a Gaussian transversally incident beam by inhomogeneous fibers. In it, a fiber formed by an arbitrary number $M$ of concentric layers was assumed and defined in the cross section of the fiber with variable radius $R_{M}$. This would be the type of limit considered in the current study, with $R_{M}=$ $\left(R_{1}^{2}-X_{1}^{2}\right)^{1 / 2}$, as displayed in Fig. 2 . The scattering amplitude could be obtained eventually by integration of $T_{\text {eik }}{ }^{(1)}\left(\mathbf{K}^{\prime}, \mathbf{K}, X_{1}\right)$ for all possible values of $X_{1}$.

The intensity distribution of the scattered field is given by

$$
\begin{aligned}
|E(\boldsymbol{\rho})|^{2}= & 1+\frac{\left|T^{(1)}\left(\mathbf{K}^{\prime}, \mathbf{K}, X_{1}\right)\right|^{2}}{|\boldsymbol{\rho}|} \\
& +\frac{T^{*(1)}\left(\mathbf{K}^{\prime}, \mathbf{K}, X_{1}\right)}{|\boldsymbol{\rho}|^{1 / 2}} \exp (-i|\mathbf{K}||\boldsymbol{\rho}|) \exp (i K \rho) \\
& +\frac{T^{(1)}\left(\mathbf{K}^{\prime}, \mathbf{K}, X_{1}\right)}{|\boldsymbol{\rho}|^{1 / 2}} \exp (i|\mathbf{K} \| \boldsymbol{\rho}|) \exp (-i K \boldsymbol{\rho})
\end{aligned}
$$

where $\rho$ represents the position vector located at the transverse plane of the fiber.

The normalization given by Eq. (2) also holds for Eq. (8), and

$$
K \rho=|\mathbf{K}||\boldsymbol{\rho}| \cos \phi,
$$

where $\phi \equiv(\mathbf{K}, \boldsymbol{\rho})$, as shown in Fig. 3 .

By equating the real and imaginary parts, we can rewrite Eq. (8) as

$$
\begin{aligned}
& \frac{\left|T^{(1)}\left(\mathbf{K}^{\prime}, \mathbf{K}, X_{1}\right)\right|^{2}}{2|\mathbf{\rho}|^{1 / 2}} \\
& \quad=T^{(1, i)}\left(\mathbf{K}^{\prime}, \mathbf{K}, X_{1}\right) \sin [K \rho(1-\cos \phi)] \\
& \quad-T^{(1, r)}\left(\mathbf{K}^{\prime}, \mathbf{K}, X_{1}\right) \cos [K \boldsymbol{\rho}(1-\cos \phi)] .
\end{aligned}
$$

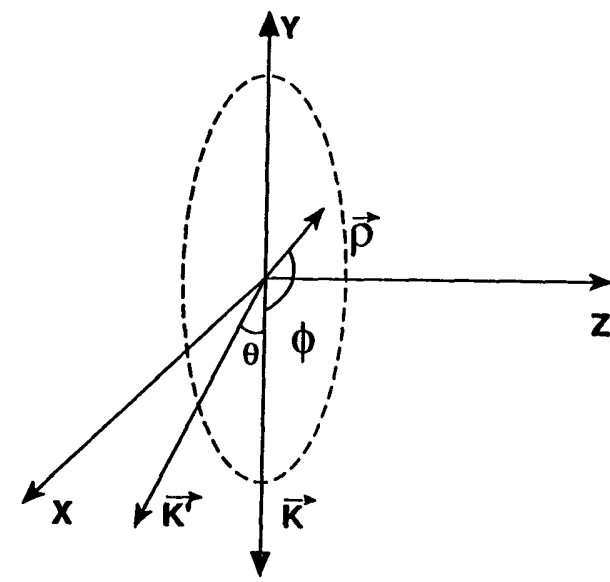

Fig. 3. Definition of the scattering angle $\theta=\left(\mathbf{K}^{\prime}, \mathbf{K}\right)$ and angle $\phi=$ $(\mathbf{K}, \boldsymbol{\rho})$, as used in Eq. (9). $\boldsymbol{\rho}$ is, in general, a three-dimensional arbitrary vector position. In the present case we assume a more simple geometry, and $|\rho|=\left(X^{2}+Y^{2}\right)^{1 / 2}$ is derived from the condition that the scatterer has cylindrical symmetry.

Equation (10) gives the analytical expression of the coupling condition. It should be noted that this expression was obtained through a normalization condition for the amplitude distribution scattered by the optical fiber in sectorial contributions.

\section{Numerical Estimates}

The numerical behavior of Eq. (10) can be determined by making a substitution for $T^{(1, r)}$ and $T^{(1, i)}$, as given in Eqs. (6) and (7), and by using a refractive-index profile for the fiber that determines the form of the eikonal phase $\varphi_{\text {eik. }}$.

Assume a parabolic refractive-index profile. ${ }^{7}$ In Fig. 4 the behavior of the phase is reproduced in the $\left[T^{(1, i)}, T^{(1, r)}\right]$ plane. As expected, a well-fitted modulation with a parabolic distribution is obtained. The variation of the phase of the complex signal, $\tan ^{-1}\left[T^{(1, i)} / T^{(1, r)}\right]$, inside the core is displayed in Fig. 5 .

In these numerical estimates, a limitation arises because of Glauber's approximation. ${ }^{11}$ If we assume that the incoming radiation has a wavelength of 0.8 $\mu \mathrm{m}$ and a core diameter of $2 \mu \mathrm{m}$, we find that

$$
\theta \ll 0.24 \mathrm{rad} \cong 14^{\circ} \text {. }
$$

This represents an upper bound for which the eikonal approximation is valid. Although a small angle is not a wide angle, the holocoupler/fiber device is working for small numerical aperture values because of the small diameter and weakly guiding approximation of the fiber. For example, values of angular distribution between to $2.5^{\circ}$ and $6^{\circ}$ (for different fiber lengths) have been reported in the literature. ${ }^{13}$ The maximum deviation of $14^{\circ}$ in the scattering angle has been assumed in the region

$$
0 \leq\left|X_{2}\right| \leq R_{1} \text {. }
$$

The numerical solution of Eq. (10) is shown in Fig. 6. Because of the presence of the term $|\rho|^{1 / 2}$, both positive and negative values are applicable. The 


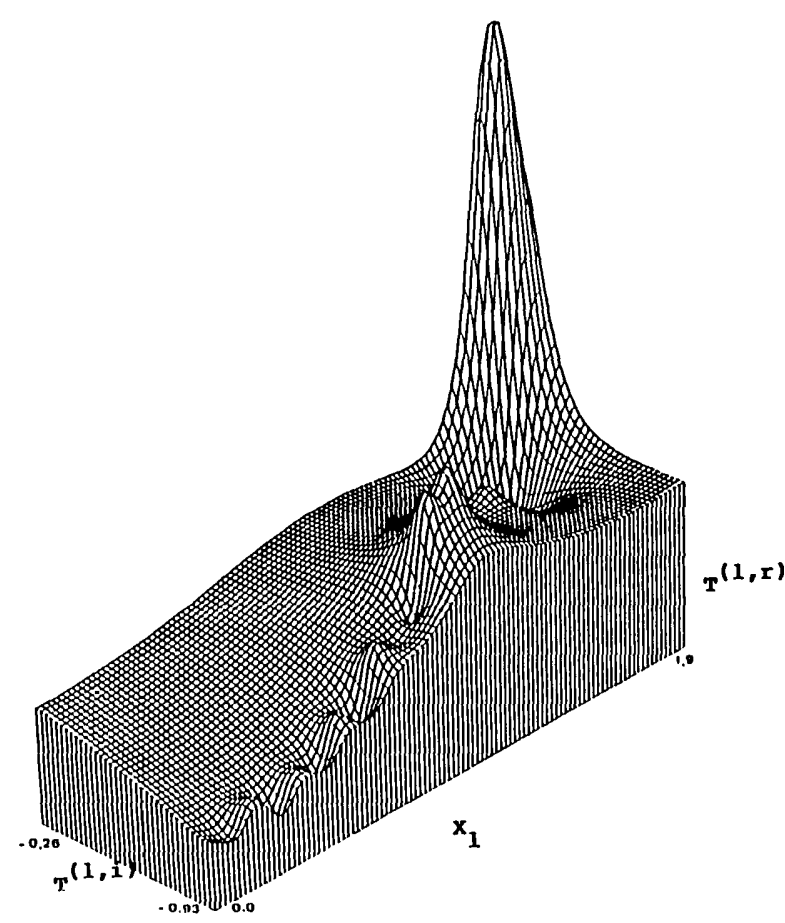

Fig. 4. Analytical behavior of the eikonal scattering amplitude. The phase profile follows the quadratic refractive-index distribution.

negative solution does not reach the coupling condition. For the positive solution, the left-hand side and the right-hand side of Eq. (10) have been numerically analyzed for different positions inside the core. For $X_{1}=0.7 \mu \mathrm{m}$, there is a crossing point that represents the position at which all the energy diffracted by the holocoupler system is confined inside the fiber. The critical aspect of this condition from the experimental point of view is related to the alignment of the system. A deviation of the order of

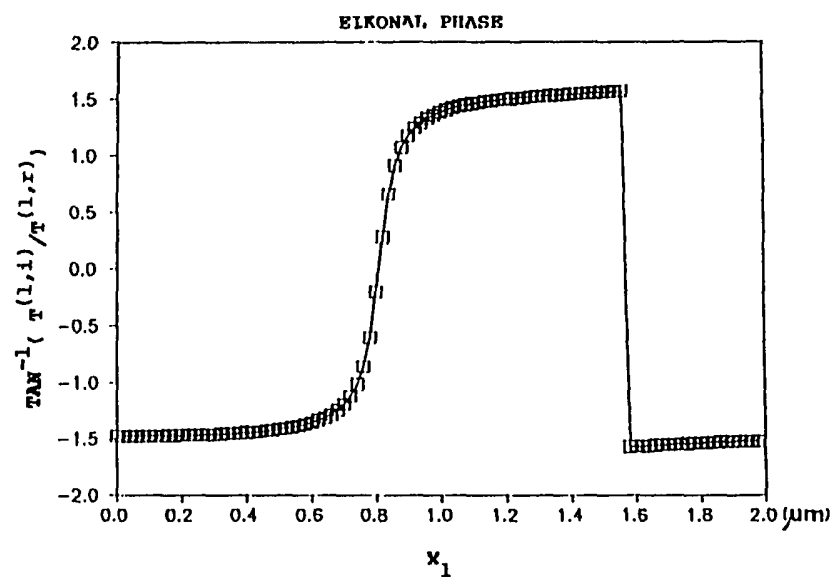

Fig. 5. Phase term $\tan ^{-1}\left[T^{(1, i)} / T^{(1, r)}\right]$ of the modified scattering amplitude [Eqs. (6 and 7)] for points inside the core of the fiber. One complete cycle is observed in the $0.0<X_{1}<1.6$ $\mu \mathrm{m}$. (The symbol $\square$ denotes the values that were numerically checked).
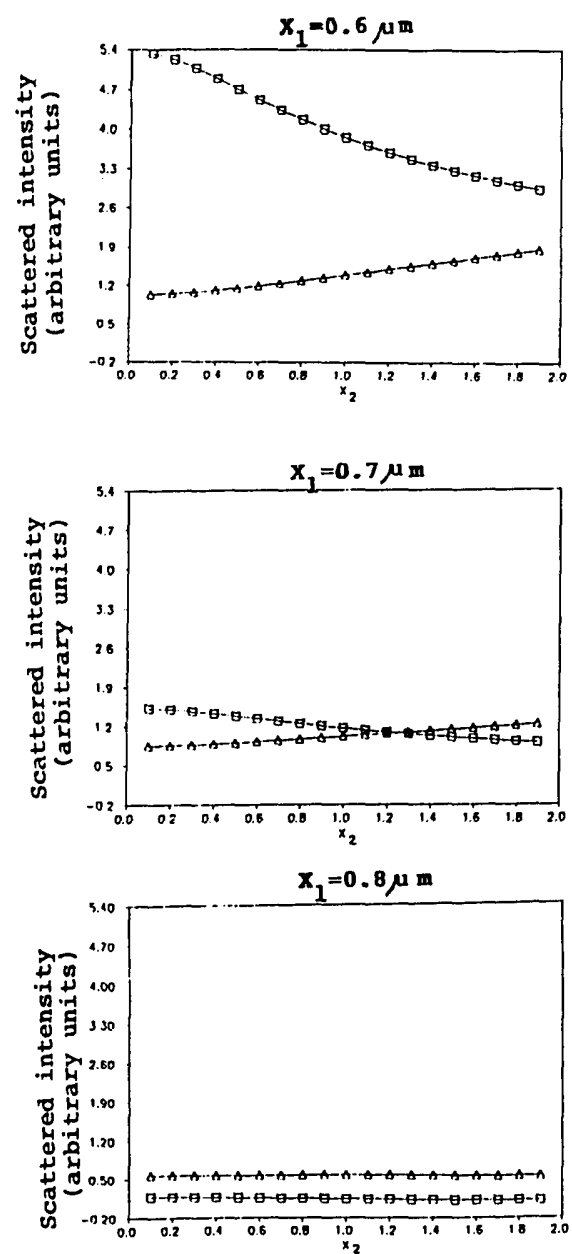

Fig. 6. Numerical solution of the coupling equation [Eq. (10)]: $\square$, left-hand side; $\Delta$, right-hand side. Three different possitions inside the core have been checked: $X_{1}=0.4,0.7,0.8 \mu \mathrm{m}$. In all cases the two terms converge monotonically and reach a single crossing point, which represents the coupling in the system for $X_{1}=0.7 \mu \mathrm{m}$ (inside the core).

$0.1 \mu \mathrm{m}$ in the signal would result in the failure of the light coupling.

\section{Double-Image Holocoupler: Coupling Condition and Linear Behavior}

The method proposed for a double-image holocoupler is displayed in Fig. 7. As observed, the recording process is similar to that for a single-image holocoupler. In order to obtain the effect of the real and the virtual images, some modifications must be introduced in the reconstruction process. In Fig. 7 we observe the experimental setup. Two mirrors $M_{1}$ and $\mathrm{M}_{2}$ are located between plate 2 to collect the second image (virtual) formed by this hologram (the symmetrical of the first-order diffracted wave with respect to the zero-order diffraction). The configuration would be similar to an optical resonator with three first-order diffracted waves generated by plates 1 (one wave front) and 2 (two wave fronts). The mirrors between the holographic plates result in a passively resonated hologram ${ }^{14}$ and aim at increasing the light-hologram interaction optically during recon- 


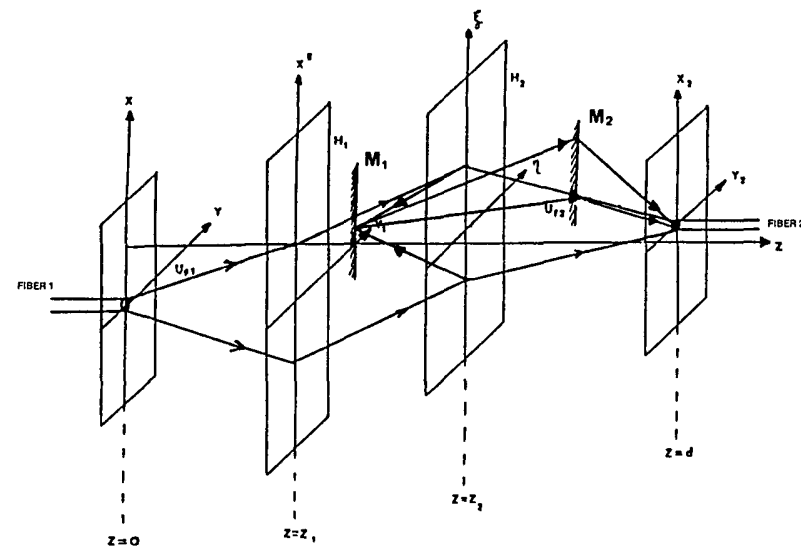

Fig. 7. Schematic representation of the experimental setup for a double-image holocoupler. The recording process is similar to the single-image case. In the reconstruction, some modifications are introduced by inserting mirrors to capture the virtual image.

struction. The two spots of light (real and virtual foci) are used to search for a larger bound in the values of the phases of the scattered waves. This would result in an optimized device. This modification implies a new formulation for the coupling condition. In the recording process the transmittance amplitude of the first hologram is

$$
T_{1}=\left|U_{F 1}+U_{R 1}\right| \cong U_{R 1} U_{F 1}^{*},
$$

and, according to the reconstruction process, the transmittance amplitude of the second hologram is

$$
T_{2}=\left|U_{F 2}+U_{R 2}\right| \cong U_{R 2} U_{F 2}{ }^{*}+U_{R 2}{ }^{*} U_{F 2} \text {. }
$$

In Eq. (13) we have introduced the effect that is produced by considering the sum of the real and virtual images generated by the second hologram. As explained in Section 2, the coupling is produced when the field transmitted by the second hologram equals the conjugate of the field scattered by the second fiber. Assuming the additional condition that the reference wave of the first recording equals the conjugate of the reference wave of the second one, the coupling condition reads ${ }^{7}$

$$
\left(U_{R 2}{ }^{*}\right)\left|U_{F 1}\right|^{2} U_{F 2}=\left(1-\left|U_{R 2}\right|^{2}\left|U_{F 1}\right|^{2}\right) U_{F 2}{ }^{*},
$$

where $U_{R 2}$ and $U_{R 2}{ }^{*}$ are the reference wave of the second recording and its conjugate, respectively, and $U_{F 1}$ and $U_{F 2}$ are the fields scattered by the first and the second fibers, respectively. $U_{F 1}$ and $U_{F 2}$ are both expressed in terms of the modified eikonal complex scattering amplitude, whose real and imaginary parts are given by Eqs. (6) and (7) along with the behavior of the eikonal phase, as shown in Fig. 4. The two reference waves $U_{R 1}$ and $U_{R 2}$ are plane-polarized monochromatic waves. The coupling equation can be expressed as

$$
F\left(X_{21}, X_{22}\right)+i G\left(X_{21}, X_{22}\right)=0,
$$

where

$$
\begin{aligned}
F\left(X_{21}, X_{22}\right) & \\
= & 2 R^{2}\left\{1-\cos \left[\varphi_{1}\left(X_{21}\right)\right]\right\}\left\{\cos \left[-\varphi_{2}\left(X_{22}\right)+2 \mathbf{k}_{r} \cdot \mathbf{r}\right]\right. \\
& \left.-\cos \left(2 \mathbf{k}_{r} \cdot \mathbf{r}\right)+\cos \left[\varphi_{2}\left(X_{22}\right)\right]-1\right\} \\
& -\cos \left[\varphi_{2}\left(X_{22}\right)\right]+1, \\
G\left(X_{21},\right. & \left.X_{22}\right) \\
= & 2 R^{2}\left\{1-\cos \left[\varphi_{1}\left(X_{21}\right)\right]\right\}\left\{\sin \left[-\varphi_{2}\left(X_{22}\right)+2 \mathbf{k}_{r} \cdot \mathbf{r}\right]\right. \\
& \left.-\sin \left(2 \mathbf{k}_{r} \cdot \mathbf{r}\right)+\sin \left[\varphi_{2}\left(X_{22}\right)\right]\right\}-\sin \left[\varphi_{2}\left(X_{22}\right)\right] .
\end{aligned}
$$

From Eq. (15),

$$
\begin{aligned}
& F\left(X_{21}, X_{22}\right)=0, \\
& G\left(X_{21}, X_{22}\right)=0
\end{aligned}
$$

Equations (18) and (19) form a pair of implicit equations in the $X_{i j}$ coordinate. A nonzero Jacobian would result in a real solution for the system.

\section{Validity of the Coupling Equation for the System}

The solution for the system represents a line of intersection between the two surfaces [Eqs. (18) and (19)]. By fixing one coordinate, we can interpret that the tangents to both surfaces at this particular point are not parallel.

From Eq. (18) we obtain

$$
\varphi_{1}\left(X_{22}\right)=\cos ^{-1}\left(a_{r 1}\right)
$$

and from Eq. (19) we obtain

$$
\varphi_{1}\left(X_{22}\right)=\cos ^{-1}\left(a_{r 2}\right),
$$

where

$$
\begin{aligned}
a_{r 1}= & {\left[1+\left(\left\{1-\cos \left[\varphi_{2}\left(X_{22}\right)\right]\right\} /\left\{\cos \left[\varphi_{2}\left(X_{22}\right)\right] \cos \left(2 \mathbf{k}_{r} \cdot \mathbf{r}\right)\right.\right.\right.} \\
& +\sin \left[\varphi_{2}\left(X_{22}\right)\right] \sin \left(2 \mathbf{k}_{r} \cdot \mathbf{r}\right)-\cos \left(2 \mathbf{k}_{r} \cdot \mathbf{r}\right) \\
& \left.\left.\left.+\cos \left[\varphi_{2}\left(X_{22}\right)-1\right]\right\}\right)\right], \\
a_{r 2}= & \left\{1-\left[1 /\left(-\cos \left(2 \mathbf{k}_{r} \cdot \mathbf{r}\right)+\left\{\cos \left[\varphi_{2}\left(X_{22}\right)\right] \sin \left(2 \mathbf{k}_{r} \cdot \mathbf{r}\right)\right.\right.\right.\right. \\
& \left.\left.\left.\left./ \sin \left[\varphi_{2}\left(X_{22}\right)\right]\right\}-\left\{\sin \left(2 \mathbf{k}_{r} \cdot \mathbf{r}\right) / \sin \left[\varphi_{2}\left(X_{22}\right)\right]\right\}+1\right)\right]\right\},
\end{aligned}
$$

$a_{r 2}= \pm a_{r 1}$.

Equation (24) implies a bound for all possible values of the phase of the planar reference wave.

Another condition for obtaining a real solution for the system is

$$
a_{r 1}, a_{r 1} \leq 1 \text {. }
$$

We have made numerical calculations for $\mathbf{k}_{r} \cdot \mathbf{r}=1$ and $\varphi_{2}$ ranging from 0 to $600 \mathrm{rad}$. The real solution given in Eq. (24) is reached at specific intervals. Some points for which there is a deviation from the 


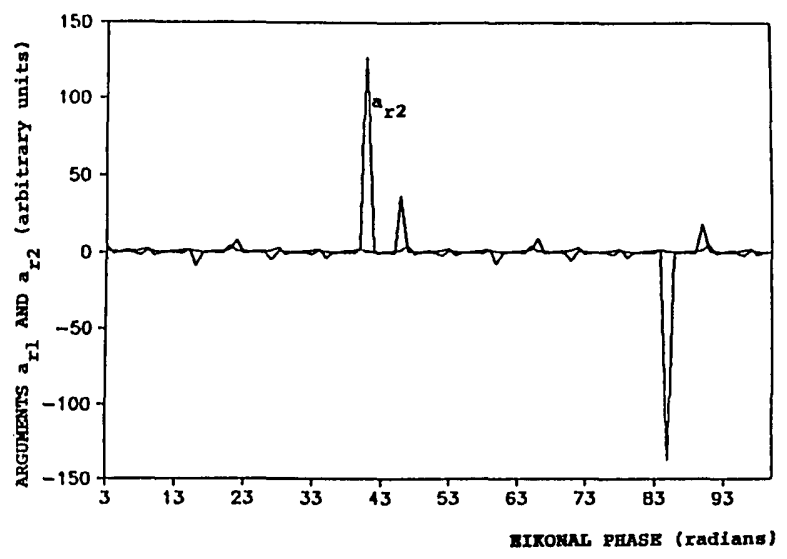

Fig. 8. Distribution shapes of arguments $a_{r 1}$ [Eq. (22)] and $a_{r 2}$ [Eq. (23)] versus eikonal phase values (in radians). The crossing point (coupling) occurs as $a_{r 1}$ equals $a_{r 2}$. The noticeable peaks correspond to argument $a_{r 2}$. Arbitrary units are represented in the vertical axis.

real definition domain have been avoided. There is a periodic behavior for two arguments $a_{r 1}$ and $a_{r 2}$ near \pm 1 (Figs. 8 and 9). By representing the two curves given by Eqs. (18) and (19) in such intervals of the real definition domain, we obtain the intersection points. In those situations the tangents to both curves are not parallel (Fig. 10). Such intervals are multiples of $2 \pi$ plus an oscillation; the value not displayed here is near the $(3.9,5.9)$ radians. In the shown cases the cross points are located in the following ranges: $11.4-$ 11.6, 162.2-162.4, 206.0-206.4, and 206.8-207.2 rad.

\section{Linear Behavior of the System}

It is important to analyze the linear behavior of the double-image holocoupler because the two-mirror configuration could be considered as an optical resonator and hence generate a nonlinear response.

The possible sources of deviation from linear behavior would be due to instabilities that are intrinsic to the device and are not due to the power source (typically 1-5 mW). The Fourier analysis techniques are easily applicable to this case. The analy-

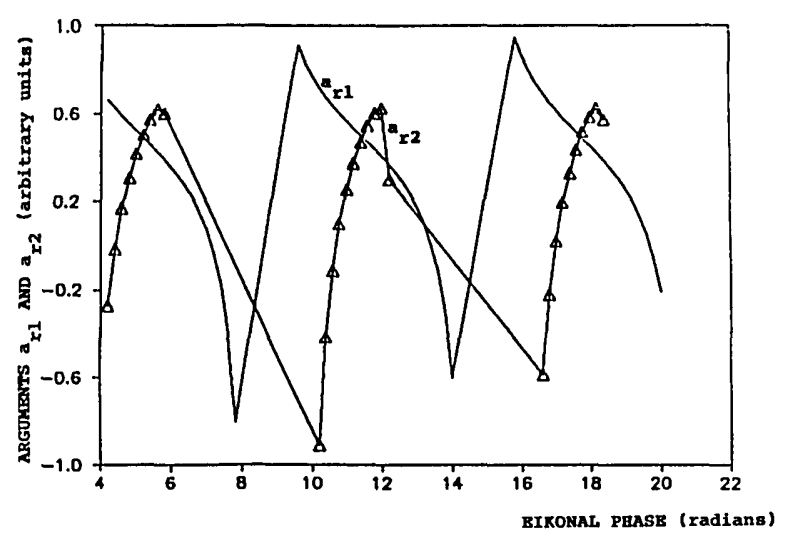

Fig. 9. Typical behavior of a phase term. Arbitrary units are represented in the vertical axis.
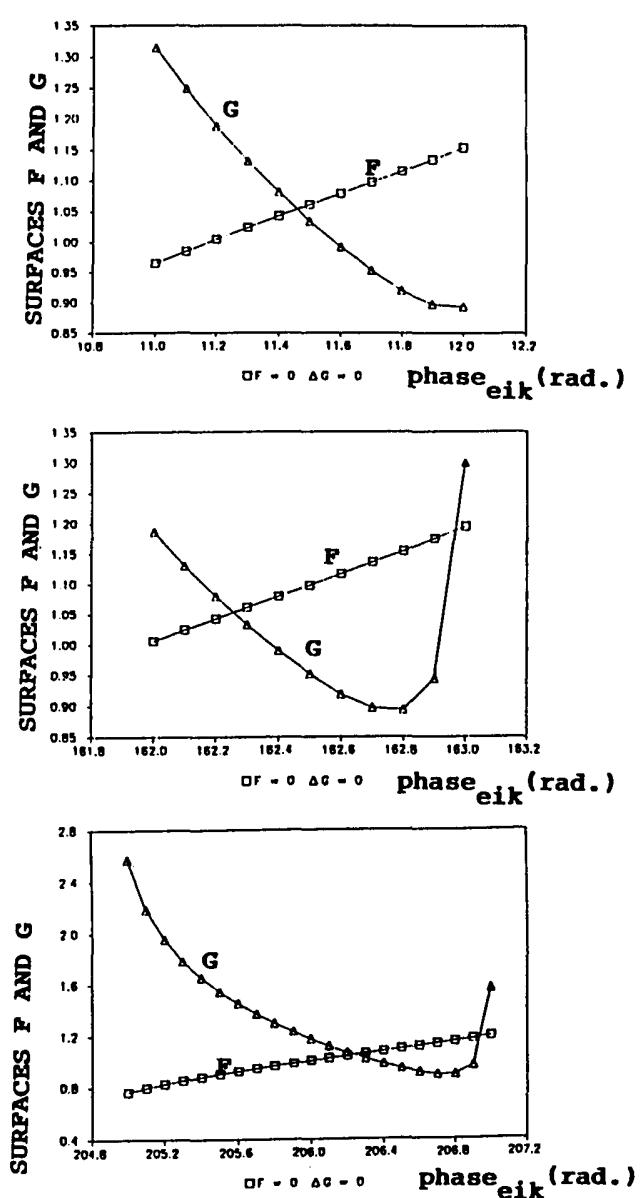

Fig. 10. Numerical solution of the coupling equation system [Eqs. (20) and (21)]. The vertical axis corresponds to $\varphi_{1}$ and the horizontal axis corresponds to $\varphi_{2}$. Three intervals for $\varphi_{2}$ have been analyzed, showing, in all cases, the intersection point representing the coupling. Values of phases are given in radians. The incoming wave incidence is $\mathbf{k}_{r} \cdot \mathbf{r}=1$.

sis of the solution in the spatial frequency domain shows that all the energy processed in this device comes from only the input of the system, i.e., the field scattered by the optical fiber.

The coupling equation (15) must be written as

$$
\mathbf{F}\left(X_{21}, X_{22}\right)=0
$$

where

$$
\begin{aligned}
& \operatorname{Re}\left[\mathbf{F}\left(X_{21}, X_{22}\right)\right]=F\left(X_{21}, X_{22}\right), \\
& \operatorname{Im}\left[\mathbf{F}\left(X_{21}, X_{22}\right)\right]=G\left(X_{21}, X_{22}\right) .
\end{aligned}
$$

Functions $F\left(X_{21}, X_{22}\right)$ and $G\left(X_{21}, X_{22}\right)$ are oscillatory functions [Figs. 11(a) and 11(b)].

The function $\mathbf{F}\left(X_{21}, X_{22}\right)$ is continuous and defined in all complex space and is Lebesgue integrable. That is,

$$
\left|\mathbf{F}\left(X_{21}, X_{22}\right)\right|^{2}=\left|F\left(X_{21}, X_{22}\right)\right|^{2}+\left|G\left(X_{21}, X_{22}\right)\right|^{2} .
$$

Also, $\left|\mathbf{F}\left(X_{21}, X_{22}\right)\right|^{2}$ is a continuous function that is 


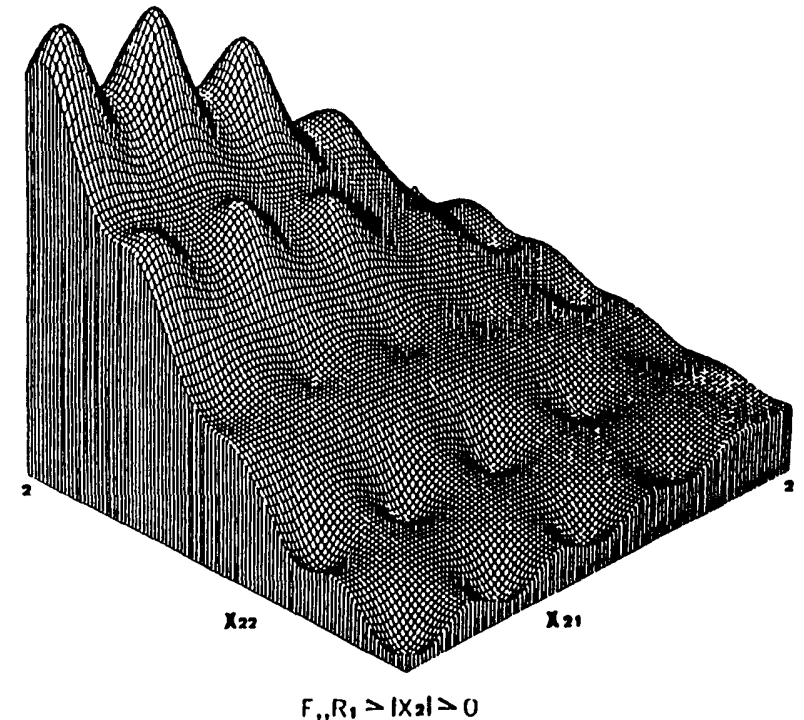

(a)

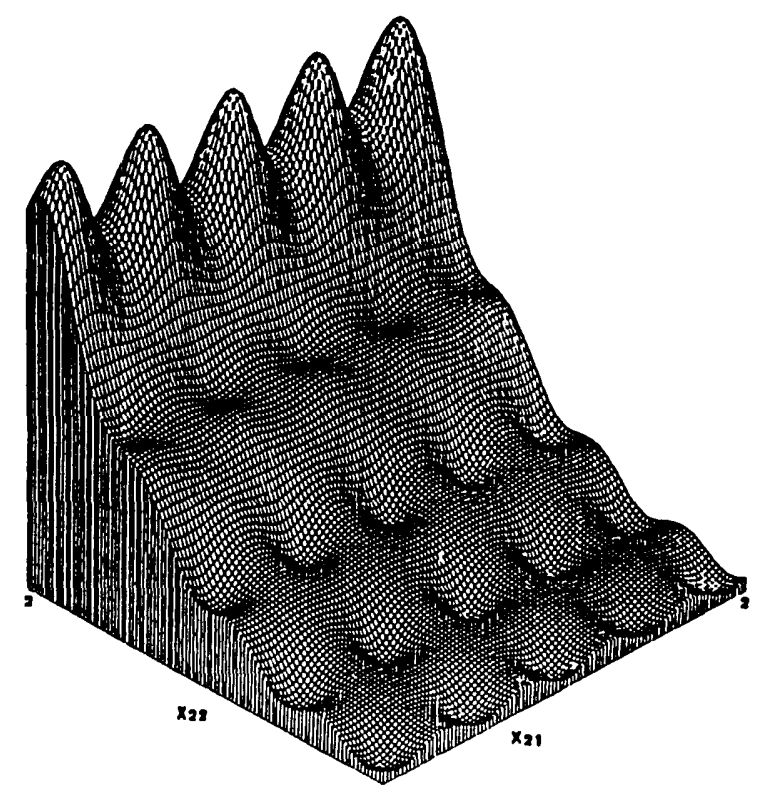

$G_{1}, R_{1}>\left|X_{2}\right|>0$

(b)

Fig. 11. (a) Three-dimensional representation of the $F\left(X_{21}, X_{22}\right)$ surface, (b) three-dimensional representation of the $G\left(X_{21}, X_{22}\right)$ surface.

defined in all real space with the property

$$
\int_{-\infty}^{\infty} \int_{-\infty}^{\infty}\left|\mathbf{F}\left(X_{21}, X_{22}\right)\right|^{2} \mathrm{~d} X_{21} \mathrm{~d} X_{22}<+\infty .
$$

By assuming the eikonal approximation for the field scattered by a fiber with a parabolic profile, ${ }^{15}$ we find that

$$
\begin{aligned}
& \varphi_{\text {eik }}=0, \quad\left|X_{2}\right|>R_{2}, \\
& \varphi_{\text {eik }}=k\left(\epsilon_{2}-1\right)\left(R_{2}{ }^{2}-X_{2}{ }^{2}\right)^{1 / 2}, \quad R_{2}>\left|X_{2}\right|>R_{1},
\end{aligned}
$$

$$
\begin{aligned}
& \varphi_{\text {eik }}= k\left(\left(\epsilon_{2}-1\right)\left[\left(R_{2}^{2}-X_{2}^{2}\right)^{1 / 2}-\left(R_{1}^{2}-X_{2}^{2}\right)^{1 / 2}\right]\right. \\
&+\left(R_{1}^{2}-X_{2}^{2}\right)^{1 / 2}\left\{\left(\epsilon_{1}-1\right)\right. \\
&\left.\left.-\left[\left(\Delta \epsilon_{1} / 3 R_{1}^{2}\right)\left(R_{1}^{2}+2 X_{2}^{2}\right)\right]\right\}\right), \\
& R_{1}>\left|X_{2}\right|>0 .
\end{aligned}
$$

Consider a Selfoc fiber with the following parameters:

$$
\begin{aligned}
R_{2} & =50.0 \mu \mathrm{m}, \\
R_{1} & =2.0 \mu \mathrm{m}, \\
\lambda & =0.8 \mu \mathrm{m}, \\
\Delta & =\left(\epsilon_{1}-\epsilon_{2}\right) / 2 \epsilon_{1}=0.01, \\
\epsilon_{2} & =2.25, \\
\epsilon_{1} & =2.3104 .
\end{aligned}
$$

The continuity for the function $\mathbf{F}\left(X_{21}, X_{22}\right)$ and its square modulus $\left|\mathbf{F}\left(X_{21}, X_{22}\right)\right|^{2}$ is guaranteed. In Fig. 12 , we observe that $\left|\mathbf{F}\left(X_{21}, X_{22}\right)\right|^{2}$ in the $R_{1}>\left|X_{2}\right|>0$ region of the fiber has a symmetrical behavior with respect to the $X_{2}=0$ axis, where $R_{1}$ is the radius of the core.

The integral of $\left|\mathbf{F}\left(X_{21}, X_{22}\right)\right|^{2}$ in the $R_{1}>\left|X_{2}\right|>0$ region gives a finite value, as expected:

$$
\begin{aligned}
& \int_{0}^{R_{1}} \int_{0}^{R_{1}} \mathrm{~d} X_{21} \mathrm{~d} X_{22}\left[\left|F\left(X_{21}, X_{22}\right)\right|^{2}\right. \\
& \left.+\left|G\left(X_{21}, X_{22}\right)\right|^{2}\right] \cong 6.351 .
\end{aligned}
$$

From the expression of the phase of the field scattered by the fiber under eikonal approximation [Eqs. (32) and (33)] and according to Eqs. (16) and (17), we deduce that the value of the integral given in Eq. (30), which is defined in the $R_{2}>\left|X_{2}\right|>R_{1}$ region, must

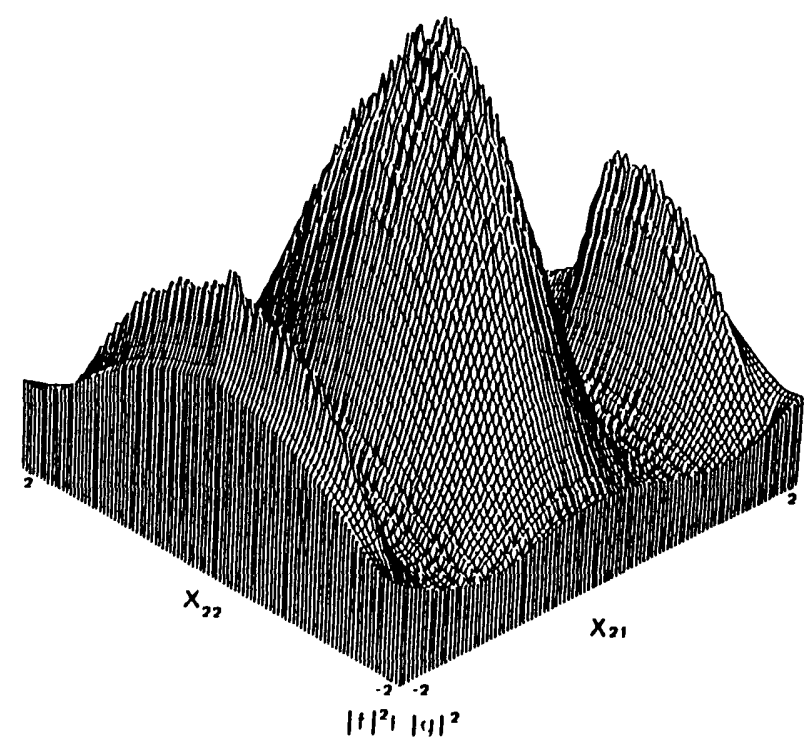

Fig. 12. Three-dimensional representation of $\left|\mathbf{F}\left(X_{21}, X_{22}\right)\right|^{2}=$ $|F|^{2}+|G|^{2}$, which shows the symmetrical behavior with respect to the origin. 


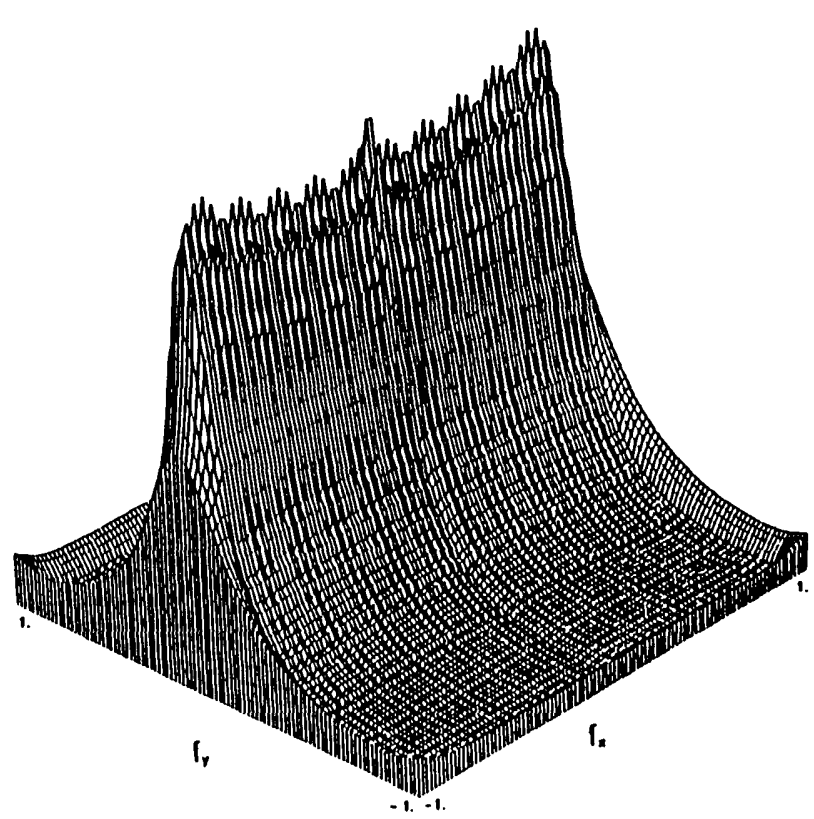

Fig. 13. Modulus of the Fourier transform of $\mathbf{F}\left(X_{21}, X_{22}\right)$ (low frequencies are represented: $\left.-1 \leq f_{x}, f_{y} \leq+1 \mu \mathrm{m}^{-1}\right)$.

be finite as well, as no singularities appear in that integral.

The Fourier transform of $\mathbf{F}\left(X_{21}, X_{22}\right)$ is expressed as

$$
\begin{aligned}
\mathscr{F}\left(f_{x}, f_{y}\right)= & \text { F.T. }\left[\mathbf{F}\left(X_{21}, X_{22}\right)\right] \\
= & \int_{-\infty}^{\infty} \int_{-\infty}^{\infty} \mathrm{d} X_{21} \mathrm{~d} X_{22} \mathbf{F}\left(X_{21}, X_{22}\right) \\
& \times \exp \left[-2 \pi i\left(X_{21} f_{x}+X_{22} f_{y}\right)\right],
\end{aligned}
$$

where F.T. is the Fourier-transform operator, $f_{x}$ and $f_{y}$ are the spatial frequencies, as an application in the

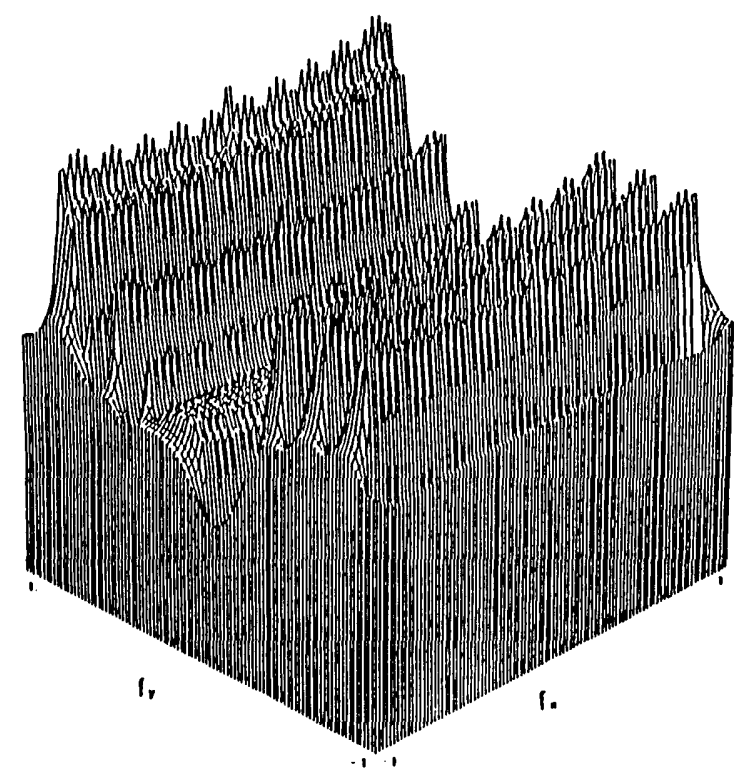

Fig. 14. Phase of the Fourier transform of $\mathbf{F}\left(X_{21}, X_{22}\right)\left(-1 \leq f_{x}\right.$, $\left.f_{y} \leq+1 \mu \mathrm{m}^{-1}\right)$.

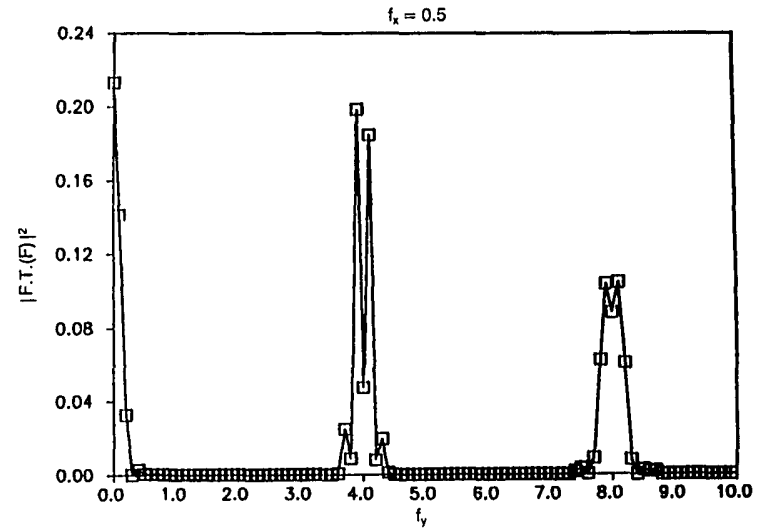

Fig. 15. Square modulus of the Fourier transform of $\mathbf{F}\left(X_{21}, X_{22}\right)$ for fixed values of $f_{x}$ (above) and fixed values of $f_{y}$ (below).

$L^{2}(R \times R)$, so the theorem of preservation of the norm is satisfied (Parseval's theorem for the scalar product of a function by itself ${ }^{16,17}$ ):

$$
\begin{aligned}
\int_{-\infty}^{\infty} \mathrm{d} f_{x} \int_{-\infty}^{\infty} \mathrm{d} f_{y} \mid & \left.\left(X_{21}, X_{22}\right)\right|^{2} \\
= & \int_{-\infty}^{\infty} \mathrm{d} X_{21} \int_{-\infty}^{\infty} \mathrm{d} X_{22}\left|\mathbf{F}\left(X_{21}, X_{22}\right)\right|^{2}
\end{aligned}
$$

We have computed the frequency spectrum of $\mathbf{F}\left(X_{21}, X_{22}\right)$ and obtained a continuous oscillatory function with spatial frequencies $f_{x}$ and $f_{y}$ (see Figs. 13 and 14).

The square modulus of the Fourier transform of functions $F\left(X_{21}, X_{22}\right)$ and $G\left(X_{21}, X_{22}\right)$ has been calculated. Symmetrical functions around the $f_{y}=0$, which oscillate with the $f_{y}$ variable (for a fixed value of $f_{x}$ ), and similar symmetrical functions around the $f_{x}=$ 0 , which oscillate with the $f_{x}$ variable (for a fixed value of $f_{y}$ ) are found.

Consider

$$
\mid \text { F.T. }\left.(G)\right|^{2}>\mid \text { F.T. }\left.(F)\right|^{2},
$$

and the contribution of the terms $\mid$ F.T. $\left.(G)\right|^{2}=f\left(f_{x}\right)$

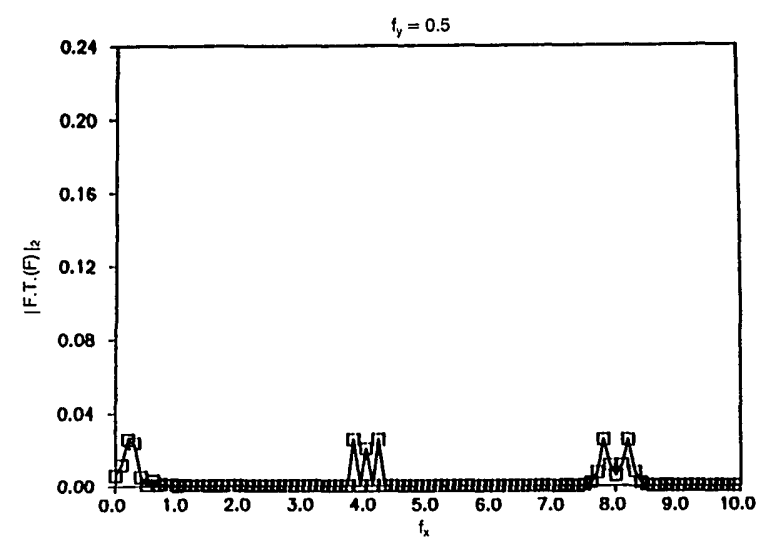

Fig. 16. Square modulus of the Fourier transform of $\mathbf{F}\left(X_{21}, X_{22}\right)$ for fixed values of $f_{y}$ (above) and fixed values of $f_{x}$ (below). 
and $\mid$ F.T. $\left.(F)\right|^{2}=f\left(f_{x}\right)$. These contributions are found to be small but different from zero (Figs. 15 and 16). This behavior of both functions, together with Eq. (36), ensures the linearity of the system.

\section{Discussion and Conclusions}

The description of the confinement of light in an optical fiber by means of a holocoupler device is mathematically expressed through a coupling equation (a normalization condition) whose numerical behavior allows one to discriminate between a singleor a double-image holocoupler. This simple interpretation arises from a formulation of the field scattered by the fiber under the eikonal approximation, where it is valid for small-angle scattering. Information on the phase distribution of the complex scattering amplitude inside the core is also obtained. This follows the refractive-index profile. Although a lateral illumination is proposed to ensure the excitation of the TM-scattered wave, which gives a lower energy conversion into guided modes than a TE one, experimental results, which are verified but not displayed here, indicate the possibility of giving a reference by the use of this particular signal. The double-image holocoupler has been also analyzed for linear behavior. The direct application of the Fourier-transform operator to the coupling equation confirms that Rayleigh's theorem holds. All possible energy losses that produce a fall in the diffraction efficiency of the holocoupler would come from the misalignement of the system with tolerances smaller than $0.1 \mu \mathrm{m}$. Of course, the absorption properties of the photomaterial should be considered as well as parts of an extended analysis of the current work.

M. L. De Pedraza thanks O. D. D. Soares for his hospitality at the Porto University. The financial support from the Comisión Interministerial de Ciencia y Tecnología (Spanish Advisory Commission for Scientific and Technological Research) is also acknowledged under project PTR 89-0162. Partial results were presented at the International Congress on Optical Science and Engineering, 11-15 March 1991, The Hague, The Netherlands.

\section{References}

1. H. Bartelt, ed., Optical Interconnections and Networks, Proc. Soc. Photo-Opt. Instrum. Eng. 1281 (1990).

2. F. Lin, "Optical holographic interconnection networks for parallel and distributed processing," in Optical Computing,
Vol. 9 of 1989 OSA Technical Digest Series (Optical Society of America, Washington, D.C., 1989), pp. 150-153.

3. O. D. D. Soares, C. Liegeois, and G. C. Righini, "Holographic interconnectors," in Optical Interconnections, O. D. D. Soares and G. C. Righini, eds., Proc. Soc. Photo-Opt. Instrum. Eng. 862, 107-114 (1987).

4. E. Schulze, "Reconfigurable optical interconnections using dynamic optoelectronic holograms," in Optical Interconnections, O. D. D. Soares and G. C. Righini, eds., Proc. Soc. Photo-Opt. Instrum. Eng. 862, 50-57 (1987).

5. M. R. Feldman, S. C. Esener, C. C. Guest, and S. H. Lee, "Comparison between optical and electrical interconnects based on power and speed considerations," Appl. Opt. 27, 1742-1751 (1988).

6. O. D. D. Soares, "Holographic coupler for fiber optics," Opt. Eng. 20, 740-745 (1981).

7. M. L. Calvo and L. De Pedraza, "Holographic coupler-optical system: mathematical model for the coupling optimization," Appl. Opt. 28, 2031-2038 (1989).

8. M. L. Calvo and L. De Pedraza, "Coupling efficiency in a holocoupler-optical fiber system," in Laser Technologies in Industry, O. D. D. Soares, ed., Proc. Soc. Photo-Opt. Instrum. Eng. 952, 301-306 (1989).

9. A. M. P. P. Leite, O. D. D. Soares, and E. Ash, "Optical-fibrebundle holographic coupler," Microwaves Opt. Acoust. 2, 45-59 (1978).

10. M. L. Calvo, P. Cheben, and M. Ulibarrena, "High efficiency off-axis holographic coupler," Opt. Commun. 88, 22-26 (1992).

11. R. J. Glauber, "High energy collision theory," in Lectures in Theoretical Physics, W. E. Brittin and L. G. Dunham, eds. (Interscience, New York, 1959), Vol. 1, p. 340.

12. R. F. Cordero Iannarella, "Transversally incident beam wave scattering from inhomogeneous fibers," J. Opt. Soc. Am. 70, 799-804 (1980).

13. E. G. Rawson, "Measurement of the angular distribution of light scattered from a glass fiber optical waveguide," Appl. Opt. 11, 2477-2481 (1972).

14. S. A. Collins, Jr., and H. J. Caufield, "Optical holographic interconnects: categorization and potential efficient passive resonated holograms," J. Opt. Soc. Am. A 6, 1568-1577 (1989).

15. R. F. Alvarez-Estrada, M. L. Calvo, and P. Juncos, "Scattering of TM waves by dielectric fibres. Iterative and eikonal solutions," Opt. Acta 27, 1367-1378 (1980).

16. L. Abellanas and A. Galindo, Introduccion a la Teoria de Operadores Lineales en Espacios de Hilbert (Universidad Complutense de Madrid, Facultad de Ciencias, Madrid, 1976); Grupo Interuniversitario de Física Teórica (GIFT), Servicio Información Científica, Facultad de Ciencias, Universidad de Zaragoza, Zaragoza, Spain.

17. A. Messiah, Mecánica Cuántica [Mechanique Quantique, (Du* nod, Paris, 1962)], translated by C. Azcárate and J. Tortella (Tecnos, Madrid, 1965), Vol. 1, App. A, p. 444. 